Thorax 1988;43:949-954

\title{
Report
}

\section{Respiratory disease in England and Wales}

\author{
From the Department of Clinical Epidemiology, National Heart and Lung Institute, London*
}

\section{Introduction}

There has been concern in recent years about the low priority given to respiratory medicine in the allocation of resources, particularly in relation to staffing ${ }^{2}$ and to research. ${ }^{3}$ It was therefore considered appropriate to assess the burden of respiratory disease in the community and the implications for the health services. This report is a synthesis of the currently available information on respiratory disease, and is focused on England and Wales; in some cases, however (notably with the General Household and the Health and Lifestyle Surveys), it has been impossible to separate the England and Wales data from those for Scotland. Throughout the paper the burden of respiratory disease has been related to all diseases combined rather than to specific disease groups.

In 1975, in a discussion on biomedical research priorities, Black and Pole published a report on the health burden imposed by 54 different disease categories. ${ }^{4}$ Although not primarily concerned with respiratory disease, they ranked it as second in importance to mental illness and mental handicap. Over the last 15 years several important changes have occurred. There has been a steady decline in the proportion of adults who smoke; in $197451 \%$ of men and $41 \%$ of women over 16 years of age were regular smokers, but by 1984 the proportions had dropped to $36 \%$ and $32 \%$. $^{5}$ There has been a continuing shift in the age distribution of the population such that the population aged 75 years or more increased by $24 \%$ from 1971 to 1981 , but that below the age of 15 fell by $13 \% .^{6}$ The incidence of some diseases (notably tuberculosis) has continued to fall whereas there have been reports

\footnotetext{
*This report by the Department of Clinical Epidemiology (which incorporates members of the Medical Research Council Cardiothoracic Epidemiology Group) at the National Heart and Lung Institute was prepared by Dr P Cullinan.

Address for reprint requests: Dr P Cullinan, Department of Clinical Epidemiology, National Heart and Lung Institute, Brompton Hospital, London SW3 6HP.

of increases both in the prevalence of and in mortality from asthma. There have also been changes in the nature and geographical pattern of air pollution and in the use of antibiotics.

Sources Data on mortality, morbidity, and financial costs are presented from the sources listed in table 1 . In each case the most recent figures available are provided.

\section{Definitions}

For the purpose of this report the following diagnostic groups, based on the ninth revision of the International Classification of Diseases (ICD), have been used so far as possible: acute upper respiratory infection including acute bronchitis and influenza (ICD 460-465, 466, 487), pneumonia (ICD 480-486), bronchitis (chronic and unspecified), emphysema and chronic airways obstruction (ICD 490-492, 496), asthma (ICD 493), malignancy of respiratory organs (ICD 160-163), respiratory tuberculosis including its late effects (ICD 10-12, 137), and other respiratory diseases (remainder of ICD codes in chapter VIII). Any deviation from these groupings is indicated in the text or tables. Congenital disease (notably cystic fibrosis) and specific infections that may affect the respiratory tract (for example, pertussis, measles) are not included.

\section{Mortality}

In 1985102449 deaths were attributed to respiratory disease in England and Wales. This was $17 \%$ of the total number of deaths from all causes- $21 \%$ of male and $14 \%$ of female deaths (table 2 ). In men almost half of all respiratory deaths were attributed to malignant disease, a substantial proportion $(29 \%)$ of which was in persons aged $45-64$ years. In women the commonest cause of respiratory death was pneumonia, accounting for half the deaths in women aged 65 years or more.

In the same year $63 \%$ of all deaths under 1 year of age were attributed to congenital lesions or to conditions specific to the perinatal period, which include several respiratory causes (respiratory distress syndrome, birth asphyxia, aspiration, etc). 
Table 1 Sources of data

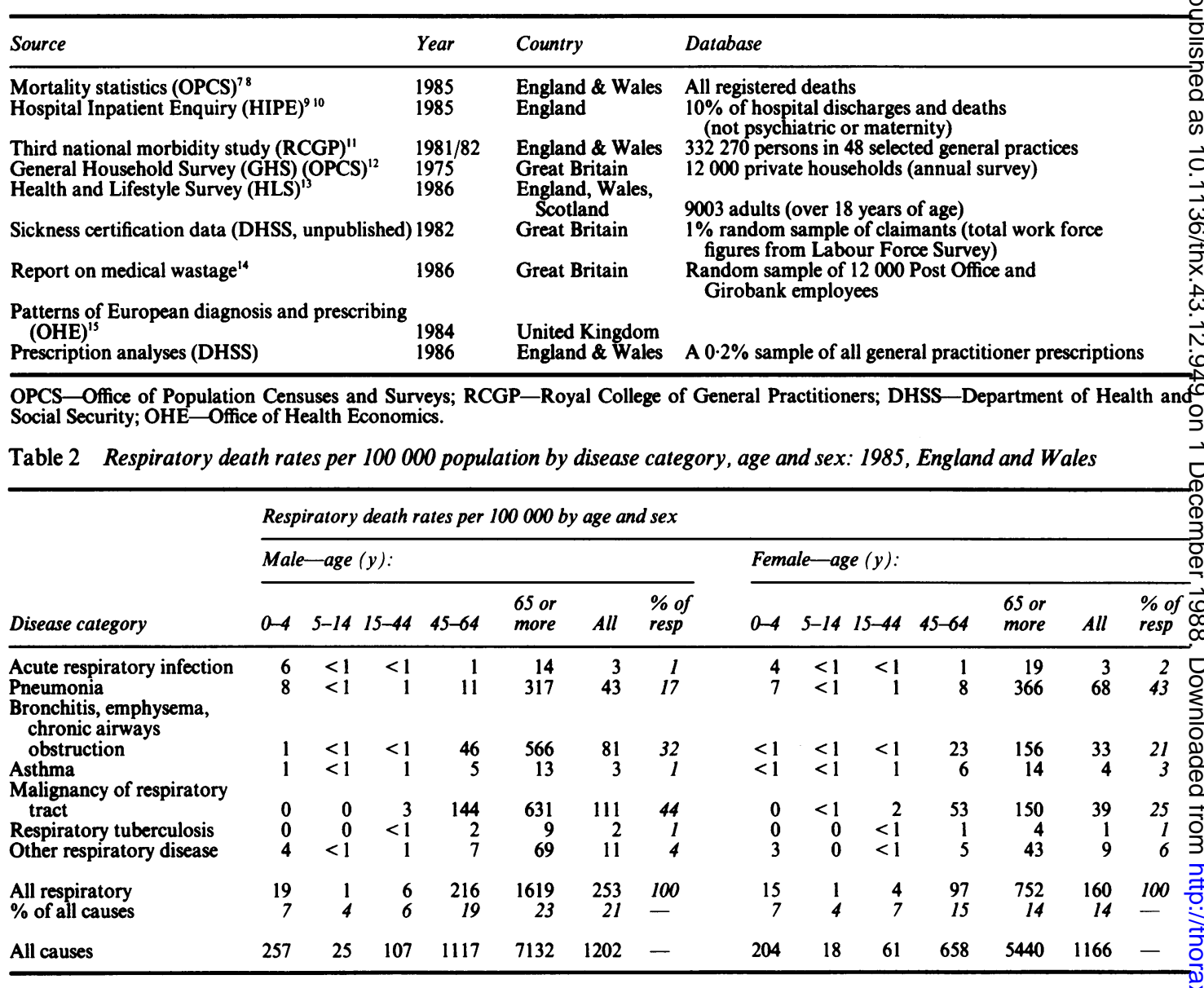

Respiratory disease (mainly acute infection or pneumonia) accounted for a further $7 \%$ of infant deaths, or about one death per 1000 live births. Apart from congenital and perinatal causes, respiratory disease was the commonest cause of infant mortality.

In each year from 1979 to 1983 respiratory disease accounted for about $21 \%$ of all deaths. In 1984 there was a change in the rules for coding death certificates, which led to a substantial reduction in the number of deaths attributed to pneumonia (from 55513 in 1983 to 24687 in 1984 and 27931 in 1985). The total number of deaths from all other respiratory causes did not alter appreciably from 1979 to 1985 , though there have been important changes in some specific categories. For example, there has been a steady decline in the number of deaths attributed to chronic bronchitis (ICD code 491) but this has been offset by a substantial increase in deaths from chronic airways obstruction (ICD code 496), so that the total number of deaths from all forms of chronic obstructive airways disease (ICD codes 490-492, 496) has actually risen. In addition, there has been a small but steady increase in: deaths from asthma.

Data from the most recent decennial supplement ${ }^{8} \delta$ show an increasing mortality with social class for all respiratory diseases (ICD chapter VIII) and for malig-o nant disease of the trachea, bronchus, and lung (ICD code 162-163). In both these categories the propor-을. tional mortality ratio in social class $\mathrm{V}$ was about twicen that in social class $I$. The difficulty in interpreting data? from the decennial supplements, due in particular to 0 lack of comparability of the information on occupation in the numerator and denominator, has been fullyo discussed elsewhere. ${ }^{16}$

\section{Morbidity}

SELF ASSESSED

General Household Survey

In $197515 \%$ of males and $16 \%$ of females interviewed for the General Household Survey reported "limiting long standing illness" (data not tabulated here). It is 
impossible to ascertain the exact proportion attributed to respiratory disease because of those reporting more than one illness; in males it must lie between $15 \%$ (the proportion reporting a single respiratory illness) and $22 \%$ (the total of those reporting disease in the three specified groups-namely, acute upper respiratory disease, bronchitis, and other lower respiratory diseases). In females the respective figures are $10 \%$ and $14 \%$. Because of the diagnostic classification used by the General Household Survey these figures do not include malignant disease or tuberculosis. The largest respiratory disease category in each sex was "bronchitis" (including acute bronchitis), which was most common in the older age groups; $6.4 \%$ of all men over the age of 65 reported themselves as chronically limited by bronchitis.

"Acute illness" was defined as that which had restricted activity in the two weeks before the survey. A little under $10 \%$ of the survey population reported such restriction $(8.7 \%$ of males and $9.8 \%$ of females). One third of acute illness in each sex was attributed to respiratory disease, about $80 \%$ affecting the upper respiratory tract; $16 \%$ reported bronchitis and $6 \%$ other lower respiratory tract diseases, including asthma.

\section{Health and Lifestyle Survey}

Six questions in the Health and Lifestyle Survey concerned chest disease. In response to a direct question, $7 \%$ of all men and $5 \%$ of all women over the age of 18 gave a history of asthma, with little variation between age groups. Similarly, $11 \%$ of both sexes reported a history of bronchitis, the proportion being considerably higher among those over $65(20 \%)$ than among those aged less than $45(7 \%)$. The proportion of people reporting persistent cough was higher in smokers, particularly in older age groups: $20 \%$ of men and $23 \%$ of women aged 65 or more who smoked regularly had a persistent cough, compared with $4 \%$ of men and $9 \%$ of women of the same age who had never smoked.

\section{GENERAL PRACTICE}

In the third national morbidity study in $1981-2,26 \%$ of males and $28 \%$ of females consulted their general practitioner for one or more respiratory complaints, the commonest being acute respiratory infection (table $3)$.

Comparison with the second morbidity study, carried out 10 years previously, ${ }^{17}$ showed a substantial increase in the rate of patients consulting for asthmafrom $9 \cdot 6 / 1000$ patients at risk in $1970-1$ to $17 \cdot 8 / 1000$ in 1981-2. Over the same period the total number of different episodes of illness attributed to asthma rose from 13.4 to $21 \cdot 4 / 1000$. This increase may be set against a decline for chronic bronchitis (ICD code 491) over the same 10 years, the rate falling from $11 \cdot 5 / 1000$ in 1970-1 to 6.0/1000 in 1981-2. Episodes attributed to chronic bronchitis also fell, from $14 \cdot 2$ to $6 \cdot 4 / 1000$.

\section{SICKNESS ABSENCE}

Work absence figures apply only to employed and insured persons and to illnesses of more than four days' duration, and thus exclude much acute respiratory illness. In 1982-3 respiratory diseases accounted for 45.5 million days of certified incapacity, $14 \%$ of all days for men and $11 \%$ of those for women. Over half the male respiratory figure was attributed to bronchitis, emphysema, or asthma, and $21 \%$ to acute respiratory infection. These two categories also accounted for over $80 \%$ of days lost by women, but in this case acute respiratory infection $(50 \%)$ was more

Table 3 Patients consulting their general practitioner by disease category, age and sex-rates per 1000 population: Royal College of General Practitioners third national morbidity study 1981-2

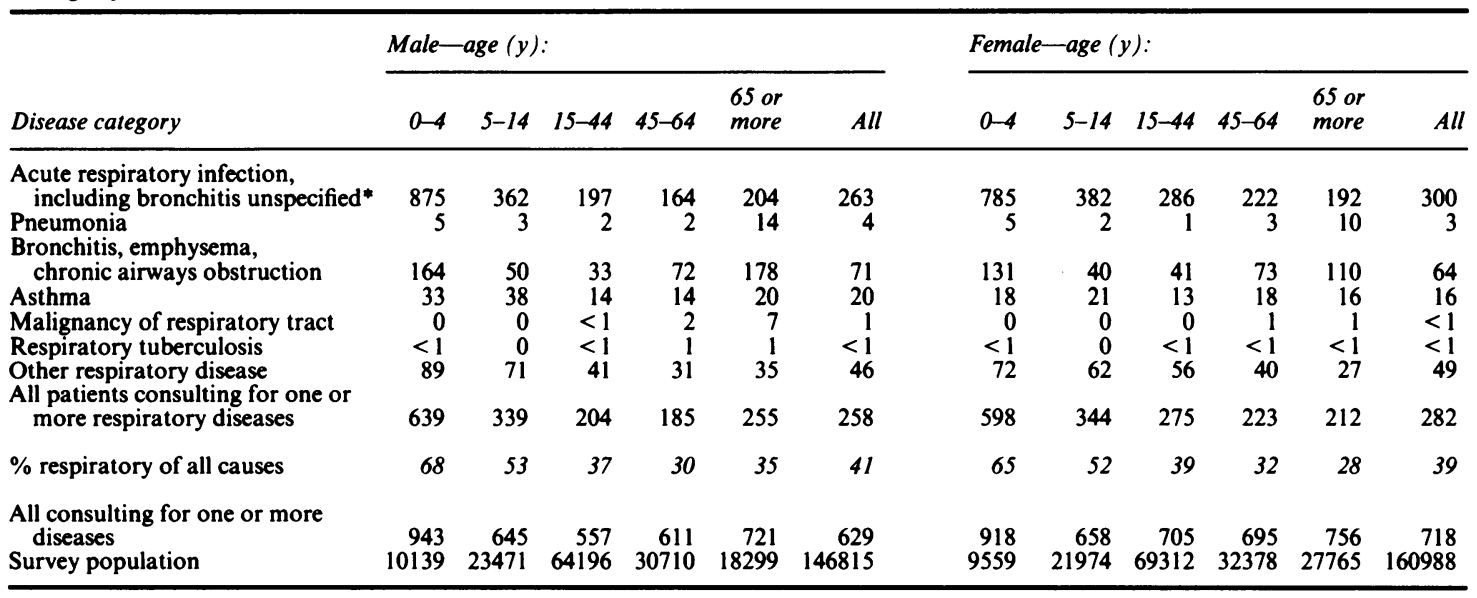

*This category includes several different diseases. 
common than bronchitis, emphysema, or asthma $(32 \%)$. Of all certified spells of incapacity, about one third in both sexes were attributed to respiratory disease.

Figures for Post Office and National Girobank employees ${ }^{14}$ show a rate of retirement on medical grounds (or death in service) due to respiratory disease of 0.9 per 1000 employees; this represents $8.0 \%$ of all male and $4.6 \%$ of all female medical retirements.

\section{Impact on the Health Service}

\section{HOSPITAL SER VICES}

The only available data that reflect hospital admissions are based on diagnosis recorded at the time of discharge or death. In 1985 there were an estimated 525420 discharges or deaths for respiratory disease, $58 \%$ of them in males. This represents $13 \%$ of the total for males and $8 \%$ for females and over $20 \%$ for both sexes in the age range 0-14 years (table 4). Apart from "other respiratory diseases" (which include upper respiratory tract disease such as diseases of the tonsils, nasal sinuses, etc) and tuberculosis, all the categories of respiratory disease account for roughly equal proportions of the total. The most striking sex differences are in the rates for bronchitis (chronic and unspecified), emphysema and chronic airways obstruction, and malignant disease in elderly men and women; in both these categories the rate for men is over twice that for women.

From 1979 to 1985 the estimated number of hospital deaths and discharges due to respiratory diseases rose from 401430 to 525420 , but remained at about $10 \%$ of the total for all causes. Figure 1 shows the trends in rates for specific respiratory diseases; the increase for obstructive airways diseases is largely accounted for by an increase in deaths and discharges for asthma, which has more than offset a steady decline in deaths and discharges for chronic bronchitis and emphysema $\frac{\bar{\sigma}}{\bar{N}}$ (the latter two categories shown by dotted lines).

In 1985 the average number of hospital beds used daily for all respiratory diseases was 16127 or $11 \%$ of os the total available.

\section{GENERAL PRACTICE}

Data from the Third National Morbidity Study 1981-2 suggest that each general practitioner in England and Wales may expect just under 1000 ct consultations each year for respiratory diseases, or $\vec{N}$ about 20 a week (this does not include consultations $\varphi$ for non-specific symptoms such as "cough"). These $\stackrel{0}{0}$ represent $22 \%$ of all male and $17 \%$ of all female 9 consultations (data not tabulated here). About $\mathbf{7 0 \%} \overrightarrow{-}$ are for acute respiratory infection, $10 \%$ for asthma, $\mathbb{D}$

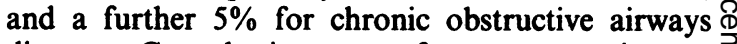
diseases. Consultation rates for acute respiratory infection are particularly high in children under $5 \mathbb{\Phi}$ years of age, for whom the rate exceeds one consulta- $\vec{\oplus}$ tion per child per year.

General practice diagnostic patterns have also been analysed in a report from the Office of Health Economics. In 1984 six of the top 20 diagnoses were respiratory, accounting together for $11 \%$ of all diagnoses. Bronchitis (unspecified, ICD code 490) accounted for $2.7 \%$ of all diagnoses and acute upper respiratory infection (ICD code 465 ) for a further $2 \cdot 1 \%$.

General practitioner prescriptions account for about $85 \%$ of all prescriptions issued in the United Kingdom. In $19829.0 \%$ of all drugs so prescribed were classified as respiratory. ${ }^{15} \mathrm{~A}$ further $9.5 \%$ were broad spectrum antibiotics, of which many were $\underset{0}{x}$ probably for respiratory infections. Unpublished 3 figures from the Department of Health and Social

Table 4 Hospital discharges and deaths: rates per 100000 population by age and sex, 1985 (Hospital Inpatient Enquiry: England)

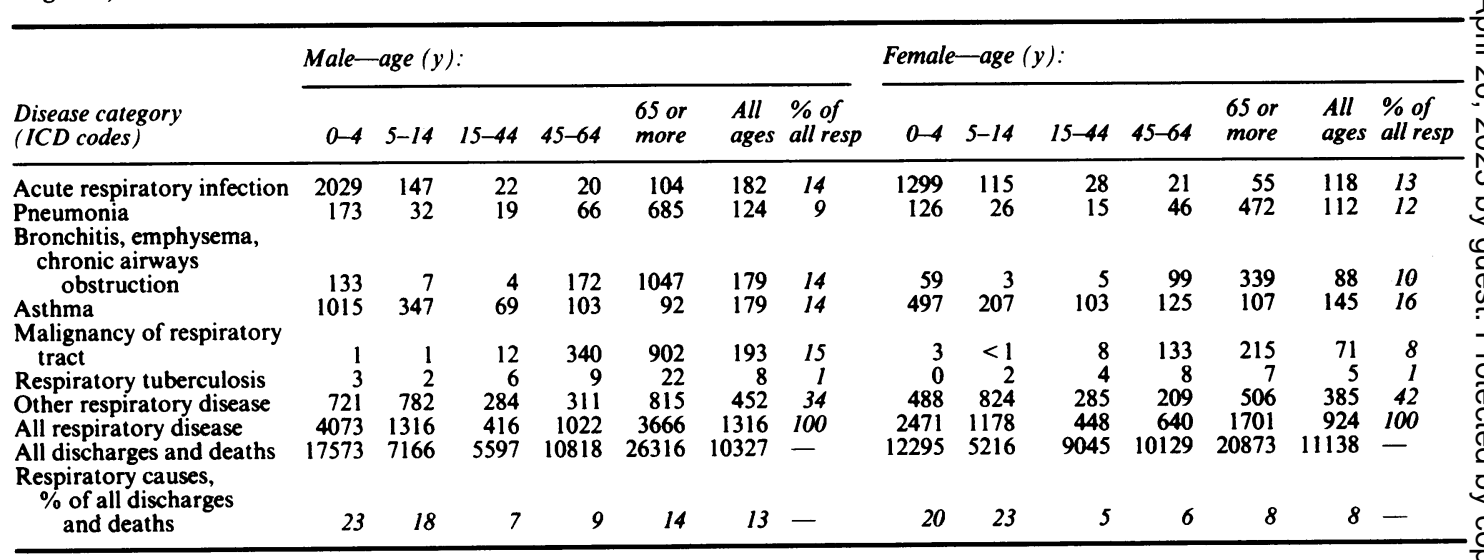




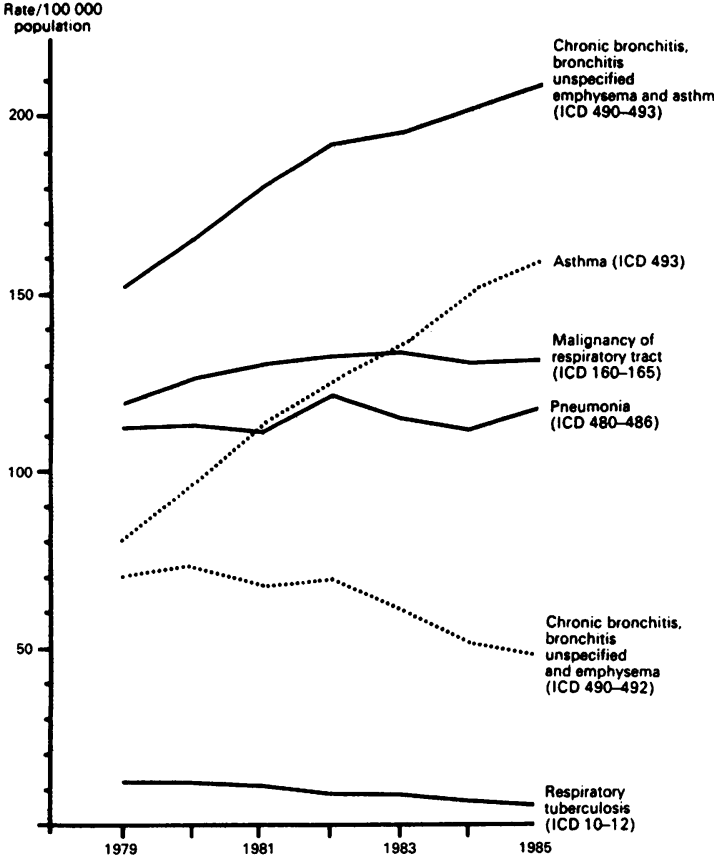

Fig 1 Hospital discharges and deaths for respiratory disease for England (Hospital Inpatient Enquiry 1979-1985). The dotted lines represent separately the rates for asthma and for the other diseases that make up ICD codes 490-492.

Security indicate that prescriptions for drugs used in the treatment of asthma rose from $3.6 \%$ of the total in 1977 to $6.0 \%$ in 1986 . In 19772.57 million prescriptions were issued for inhaled bronchodilators $(0.7 \%$ of the total); by 1986 the figure had risen to 10 million $(2.9 \%$ of the total). Over the same period prescriptions for theophylline preparations rose from 2.38 million to 3.82 million.

\section{Costs}

In 1984 the total health expenditure in the United Kingdom was about $£ 17.3$ billion. ${ }^{18}$ Hospital services accounted for most of this ( $£ 10.28$ billion), general practitioner services accounting for $£ 1.23$ billion, pharmaceutical expenditure $£ 1.75$ billion, and miscellaneous services (ambulances, administration, etc) a further $£ 2.06$ billion. The data presented in this paper suggest that about $10 \%$ of all hospital expenditure, pharmaceutical services, and miscellaneous services and $20 \%$ of general practitioner services are for respiratory disease. Thus the total health expenditure on respiratory disease in the UK in 1984 was about $£ 1.66$ billion, or $£ 30$ per person per year-a little less than $10 \%$ of the overall health expenditure.

\section{Discussion}

Respiratory disease clearly remains an important cause of death and of both acute and chronic sickness. In England and Wales in 1985 it accounted for one in five of all deaths in men, and for one in eight of all deaths in women; most of these were from lung cancer, chronic obstructive airways diseases, and pneumonia. In the same year $10 \%$ of all hospital admissions $(20 \%$ in children under 14 years) were due to respiratory disease. In 1981-2 about one in five of all general practice consultations were for respiratory disease and in 1982 at least 45 million working days were lost on account of it (13\% of the total).

This study focuses on respiratory disease and no systematic attempt has been made to rank the resultant health burden against other disease categories. Some comparisons, however, may be interesting. In the age group 45-64 years respiratory malignancy accounted for $13 \%$ of all male deaths in 1985 , compared with $39 \%$ from ischaemic heart disease (ICD codes 410-414). In the National Morbidity Survey of $1981-2$ about $27 \%$ of all registered patients consulted for respiratory diseases ( $2 \%$ for asthma), compared with $13 \%$ for musculoskeletal and connective tissue diseases $(3 \%$ for back pain) and less than $2 \%$ for ischaemic heart disease. In $19868 \%$ of Post Office medical retirements (or deaths in service) in men were attributed to respiratory disorders; $20 \%$ were due to ischaemic heart disease, and $29 \%$ to musculoskeletal disease.

These data have been collected from various sources, in some cases from large national surveys and in others from routinely collected statistics. Difficulties in interpretation inevitably arise, particularly in the comparison of data obtained from different sources at different times. The accuracy of diagnostic categories is likely to vary considerably between the various sources and is likely to be least reliable for self reported illness. Self assessed morbidity from the General Household Survey has, however, been found to correlate well with morbidity as assessed by general practitioners ${ }^{19}$; and in general there is good agreement between the various sources. In some cases detailed interpretation of data is difficult; hospital admission data, for example, do not distinguish hospital discharge from inpatient death or the number of patients admitted from the total number of admissions. An increase in the total number of admissions may therefore reflect a change in admission policy (an increase in the number of admissions per patient) rather than an increase in the number of different patients admitted. Similarly, drug prescription data may reflect an increased disease prevalence or an increase in the number of drugs available, or a change in medical perception of a disease and its treatment. 


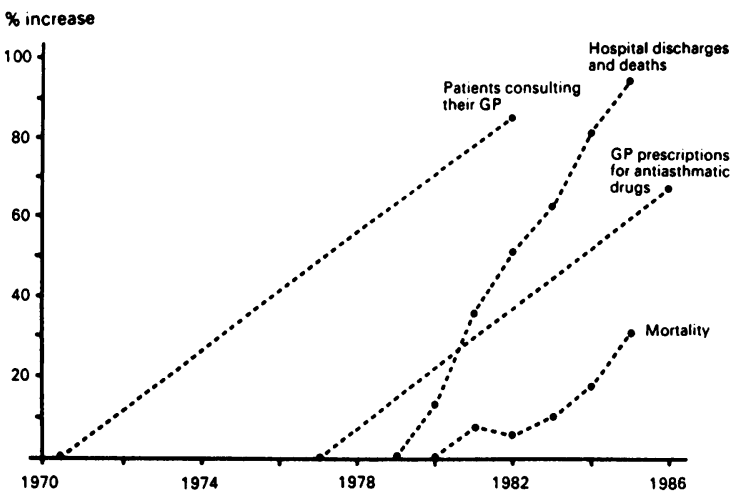

Fig 2 Changes in asthma morbidity and mortality.

Some of the most consistent trends are seen in asthma over the last 10 years (fig 2). There have been substantial increases in hospital discharges and deaths, rates of general practice consultations, drug prescription rates, and mortality ascribed to this cause. It is not possible to say how much of this is attributable to diagnostic transfer (for example, away from "chronic bronchitis" in adults or "wheezy bronchitis" in children) and how much to a real increase in the incidence or severity of asthma. Burney ${ }^{20}$ has reported an increase in asthma mortality in the 5-34 year age group, in which diagnoses are considered to be most consistent.

Although notification rates for tuberculosis continue to fall, the decline has been slower than many predicted. This is partly due to the high rates of tuberculosis in immigrants from the Indian subcontinent $^{21}$ as well as to a slowing of the rate of decline in the white population. ${ }^{22}$ It remains to be seen whether there will be further slowing of the decline in notifications or even an increase, as in the United States, where it has been attributed to the rising prevalence of human immunodeficiency virus infection. ${ }^{23}$

Over the past 10 years there has been a steady decline in the proportion of adults who smoke. If this continues, it is likely in due course to be reflected in a decline in the mortality and morbidity rates for obstructive airways diseases and, eventually, in a fall in the mortality rate for lung cancer. Over the past few years, however, mortality rates for chronic nonmalignant respiratory disease (all forms) have changed little and the small fall in mortality from respiratory cancers in men has been offset by a rise in women. Hospital discharge and death rates for chronic bronchitis and emphysema have fallen since 1979 but there has been a small increase in the rates for lung cancer.

Unpublished data from the General Household Survey of 1975 and the Hospital Inpatient Enquiry of
1985 were kindly provided by the Office of Population Censuses and Surveys and those from the Health and $\frac{\bar{\sigma}}{\bar{T}}$ Lifestyle Survey by the Health Promotion Trust $\frac{\omega^{2}}{2}$ (Dr Brian Cox). This study was assisted by a grant $\stackrel{\varnothing}{\complement}$ from the British Lung Foundation.

\section{References}

1 Citron KM, Lewis DR, Nunn AJ. Staffing in thoracic medicine. $B r$ Med J 1980;281:887-8.

2 British Thoracic Society. Thoracic medicine in Great Britain. $\vec{\omega}$ London: British Thoracic Society, 1987.

3 Green M. Funding respiratory research. Thorax 1985; 40:81-4.

4 Black D, Pole J. Priorities in biomedical research. Indices of Burden. Br J Prev Soc Med 1975;29:222-7.

5 Office of Population Censuses and Surveys. General Household Survey. 1984. London: HMSO, 1984. (Series GHS, No 14.)

6 Office of Population Censuses and Surveys. OPCS monitor. London: OPCS, 1984.

7 Office of Population Censuses and Surveys. Mortality statistics: cause. London: HMSO, 1985. (Series DH2, No 12.)

8 Office of Population Censuses and Surveys. Occupational mortality 1979-1980 and 1982-1983. London: HMSO, 1986. Ref. DS, $\overrightarrow{0}$ No. $6,1986$.

9 Office of Population Censuses and Surveys. Hospital Inpatient Enquiry (England): trends 1979-1985. London: HMSO, 1987.

10 Office of Population Censuses and Surveys. Hospital Inpatient Enquiry (England) 1985. London: HMSO, 1985.

11 Royal College of General Practitioners, Office of Population Censuses and Surveys, and Department of Health and Social $\frac{\circ}{\mathbb{D}}$ Security. Morbidity statistics from general practice 1981-82. Third national study. London: HMSO, 1986.

12 Office of Population Censuses and Surveys. General Household Survey. London: HMSO, 1975.

13 Cox BD, et al. Health and lifestyle survey. London: Health Promotion Research Trust, 1987.

14 Post Office. Report on medical wastage 1985-1987. London: Post Office, 1987.

15 O'Brien B. Patterns of European diagnoses and prescribing. London: Office of Health Economics, 1984.

16 Office of Population Censuses and Surveys. Occupational mortality. Decennial supplement 1970-1972. London: HMSO, 1978.

17 Royal College of General Practitioners, Office of Population Censuses and Surveys, and Department of Health and Social Security. Morbidity statistics from general practice 1970-71. 윽 Second national study. London: HMSO, 1974. (Studies on $D$ medical and population subjects, No 26.) 18 Office of Health Economics. Health expenditure in the UK.
London: OHE, 1986.

19 Crombie DL, Fleming DM. Comparison of second national morbidity study and the general household survey 1970-71. Health Trends 1986;18:15-8.

20 Burney P. Asthma mortality in England and Wales: evidence for a $O$ further increase 1974-84. Lancet 1986;ii:323-6.

21 Medical Research Council Tuberculosis and Chest Diseases Unit. $\stackrel{\circ}{C}$ National Survey of notifications of tuberculosis in England and $\mathbb{D}$ Wales in 1983. Br Med J 1985;291:658-61.

22 Springett VH, Darbyshire JH, Nunn AJ, Sutherland I. Changes in $\square$ Tuberculosis Notification Rates in the White Ethnic Group in England and Wales between 1953 and 1983. J Epidemiol $\underset{\mathbb{D}}{\vec{D}}$ Commun Health (in press).

23 Anonymous. Tuberculosis - United States 1985-and the possible $\mathbb{Q}$ impact of human T-lymphotropic virus type III/lymphadenopathy associated virus infection. Mortality and Morbidity Weekly Record 1986;35:74-6. 